\title{
Epidemiological study of abdominal tuberculosis among Indian migrants and the indigenous population of Leicester, 1972-1989
}

\author{
C S J Probert, V Jayanthi, A C Wicks, D L Carr-Locke, P Garner, J F Mayberry
}

\begin{abstract}
A retrospective, epidemiological study of abdominal tuberculosis in the city of Leicester from 1972 to 1989 is reported. Potential cases were identified from hospital medical records and endoscopy lists, in addition to the county notification register. The city population of 280000 included over 75000 South Asians. There were 146 cases among South Asians and six in Europeans, four of whom were British. The standardised incidence of abdominal tuberculosis in South Asians decreased significantly from 22.3 cases/10\%/year during the 1970 s to 9.2 cases/10\%/year in the $1980 \mathrm{~s}$ $\left(\chi^{2}=42, p<0.001\right)$. The incidence during the 1980 s was $10 \cdot 7 / 10^{5} /$ year in Hindus, $8 \cdot 7 / 10^{5} /$ year in Sikhs, and 4.6/10 $/$ year in Muslims. The relative risk to Hindus was 2.3 fold greater, and for Sikhs 1.9 fold greater, than that for Muslims, a finding similar to that in pulmonary tuberculosis. The standardised incidence in Europeans was $0 \cdot 2 / 10^{5} /$ year and they had significantly less abdominal tuberculosis than South Asians $(Z=8.6, p<0.001$ and relative risk=46). The standardised mortality ratio was significantly increased in Europeans (standardised mortality ratio $=755,95 \%$ confidence interval 90-2730, $\chi^{2}=11.4, p<0.001$, but not in South Asians (standardised mortality ratio $=68,95 \%$ confidence interval 20-160). Resection rates were similar between the two ethnic groups. Abdominal tuberculosis still occurs among migrants, and clinicians should remain alert to this in South Asians. (Gut 1992; 33: 1085-1088)
\end{abstract}

South Asian migrants are at greater risk of pulmonary tuberculosis than Europeans' and the incidence within various ethnic groups may differ. ${ }^{1}$ The epidemiology of abdominal tuberculosis has received little attention, however, in Britain. ${ }^{2-}$ The differentiation of abdominal tuberculosis from Crohn's disease may present specific problems in South Asians, ${ }^{5-8}$ particularly as the incidence of Crohn's disease seems to be increasing. ${ }^{9}$

Leicester is in the East Midlands and has a large textile industry. Substantial immigration occurred in the 1960s and early 1970s from South Asia and East Africa. Most migrants were of Gujarati or Punjabi descent. ${ }^{1011}$ The former are predominantly Hindu and the latter Sikh, although there are also a number of Muslims of South Asian descent in the city. 'South Asian' refers to people whose families originated in
India, Pakistan or Bangladesh, and includes those who moved to East Africa, as well as their offspring. ${ }^{1213}$

The aim of this study was to determine the epidemiology of abdominal tuberculosis in the city of Leicester and to compare the incidence and mortality in the indigenous population with that of South Asian migrants.

\section{Methods}

\section{PATIENTS}

The study was retrospectively carried out from 1 January 1972 to 31 December 1989 inclusive. Potential cases were identified from hospital activities analysis and Korner data from the Leicestershire Health Authority and individual hospitals, from the county tuberculosis notification register and personal indexes (DLCL and ACW). Demographic details and the results of laparotomy, histopathological, microbiological, radiological, and endoscopic investigations were noted. Only patients diagnosed while residing in the city during the study period were accepted into the incidence study.

Cases were included if there was (i) microbiological evidence of abdominal tuberculous infection, and/or (ii) histological evidence of caseating granulomas or mycobacteria, and/or (iii) characteristic lymphocytic ascites with a clinical response to antituberculous chemotherapy and/or (iv) radiological evidence of terminal ileal disease again with a clinical response to antituberculous chemotherapy, or (v) a high index of clinical suspicion and a clinical response to antituberculous chemotherapy.

The city of Leicester population data were obtained from a variety of sources: Office of Population Censuses and Surveys (OPCS) (1971 and 1981), ${ }^{14} 15$ Leicester Council for Community Relations (1978 and 1982), ${ }^{16}$ Survey of Leicester (1983), " and Leicester City Council projections (1990) (personal communication). The Survey of Leicester" gave the detailed structure of each ethnic group.

The date of diagnosis was used for incidence calculations. Incidence values were standardised by the direct method ${ }^{17}$ using the East Midlands whole population structure for Europeans and the Leicestershire South Asian population structure for South Asians. ${ }^{18}$ Data were analysed using a modified Cochran's test for standardised incidence values, ${ }^{19} \chi^{2}$ test, relative risk for population studies, and $95 \%$ confidence intervals. ${ }^{20}$
The outcome of disease was investigated

\section{Correspondence to: Dr C S J Probert, Leiceste General Hospital, Gwendolen Accepted for publication 6 December 1991 \\ Groby Road Hospital,}




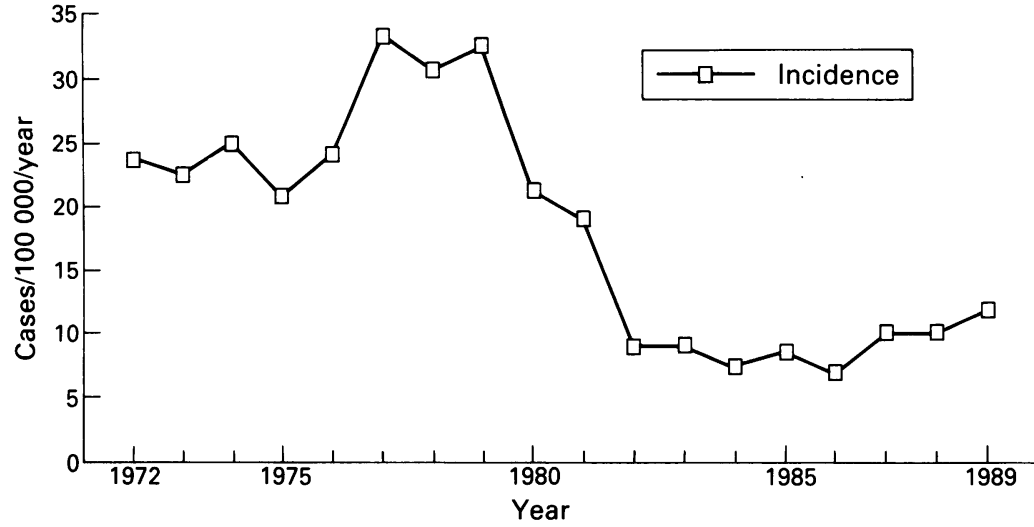

Figure 1: Incidence of abdominal tuberculosis in South Asians. Incidence peaked at the end of the 1970s and fell during the 1980 s. The rolling incidences were calculated from the mean of three consecutive years. For example 1976 value was found from data from 1975, 1976, and 1977.

through resection rates and a study of mortality in South Asians and Europeans. In Europeans the expected mortality for each patient was found by adding the risk of death each year according to current age from standardised mortality statistics for the East Midlands..$^{21}$ This was done from the year of diagnosis until 1 January 1990 or until death. For South Asians, the expected mortality was found in a similar manner, using immigrant mortality data. ${ }^{22}$ Age specific mortality was used throughout the study taking account of the increasing age, but unlike the whole population estimates used for Europeans no adjustment could be made for the changing annual mortality rates as these data were unavailable. Mortality was compared using 95\% confidence intervals using a Poisson distribution and $\chi^{2}$ test.

Ethical Committee approval was given for the study.

\section{Results}

One hundred and fifty four cases of abdominal tuberculosis were diagnosed in the city between 1972 and 1989, 146 were South Asians and six Europeans, four of whom were English, one Irish, and one Southern European. One hundred and thirty eight of the 146 South Asians with abdominal tuberculosis were born outside Britain.

\section{INCIDENCE}

The mean standardised incidence for Europeans from 1972 to 1980 was 0.09 cases $/ 10^{5} /$ year $(95 \%$ confidence interval (CI) $0-0 \cdot 19 / 10^{5} /$ year). The standardised incidence in South Asians was $22 \cdot 3$ cases $/ 10^{5} /$ year (95\% confidence interval 16-26/ $10^{5} /$ year). South Asians had significantly more incidence of abdomina tuberculosis in South Asians. There was a bimodal distribution. In the 1980s the incidence fell in each age band, particularly the young.

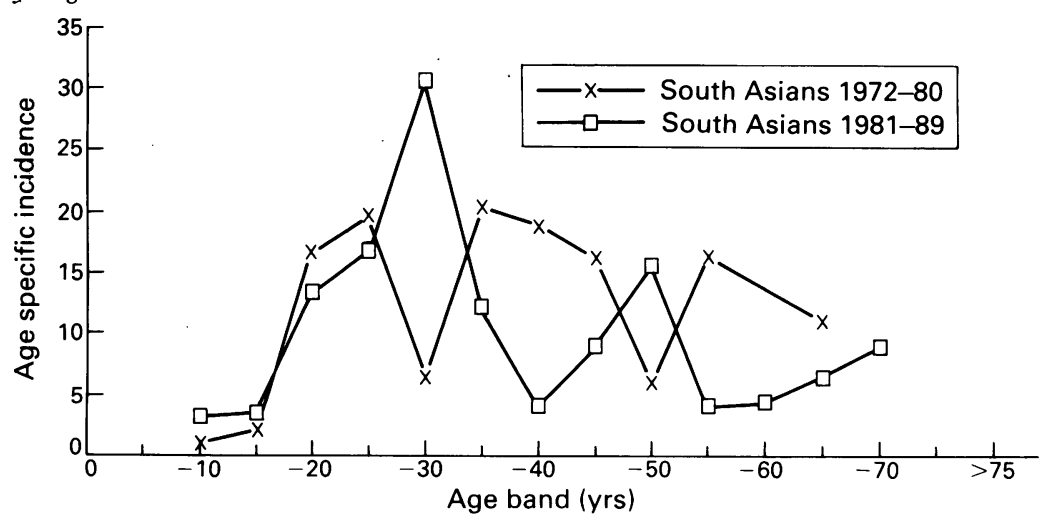

abdominal tuberculosis than Europeans in the 1970 s $(Z=5 \cdot 6, p<0.001)$ and the relative risk for South Asians was 248 (95\% confidence interval 61-1006).

From 1981 to 1989 the incidence of abdominal tuberculosis fell significantly in South Asians to $9 \cdot 2$ cases $/ 10 \% /$ year $(95 \%$ confidence interval $6 \cdot 6$ $11 \cdot 6 / 10^{5} /$ year) ( $\chi^{2}$ with Yates correction $=42$, $\mathrm{p}<0.001$ ). In Europeans the incidence was 0.2 cases $/ 10 \%$ year $(95 \%$ confidence interval $0-0 \cdot 40$ / $105 /$ year), a change which was not statistically significant $\left(\chi^{2}=0.17 \mathrm{~ns}\right)$. Although South Asians had significantly more abdominal tuberculosis than Europeans in the $1980 \mathrm{~s}(\mathrm{Z}=8 \cdot 7, \mathrm{p}<0.001)$, the relative risk to South Asians fell to $46(95 \%$ confidence interval 17-128) (Fig 1).

The proportion of cases for each South Asian religious group was the same during 1972-80 and 1981-89 $\left(\chi^{2}=0.459 \mathrm{~ns}\right)$. In the $1980 \mathrm{~s}$ the standardised incidence among Hindus was $10 \cdot 7$ cases $/ 10^{5} /$ year $(95 \%$ confidence interval $5 \cdot 0$ $11 \cdot 8 / 10^{5} /$ year) and Muslims 4.6 cases $/ 10^{5} /$ year (95\% confidence interval $2 \cdot 0-7 \cdot 0 / 10^{5} /$ year).

\section{SEX RATIO AND AGE SPECIFIC INCIDENCE}

There was a small excess of abdominal tuberculosis in male Europeans and Hindus (relative risk $5(95 \%$ confidence interval $0 \cdot 5-43)$ and $1 \cdot 27$ (95\% confidence interval $0 \cdot 9-1 \cdot 8$ ) respectively). Sikh and Muslim women, however, were at slightly greater risk than men (relative risk 1.67 (95\% confidence interval $0 \cdot 7-3 \cdot 3)$ and $1 \cdot 1(95 \%$ confidence interval $0 \cdot 4-3 \cdot 3$ ) respectively).

The age specific incidence in South Asians showed a bimodal distribution, with those over 70 years having the greatest incidence (Fig 2). The incidence fell in each age group during the study, particularly among young adults. There were too few cases among Europeans to do a comparable analysis.

\section{SITE OF DISEASE}

Of the 146 South Asian cases, 43\% had peritoneal disease, $39 \%$ intestinal tuberculous, $12 \%$ intraabdominal lymphadenopathy, and the remainder hepatic involvement. Five of the six Europeans had peritoneal tuberculosis and other hepatic disease. One European was diagnosed at necropsy after surgery.

\section{RESECTION RATE}

Of the 58 South Asian patients with intestinal tuberculosis, 26 had a resection. One of the four Europeans with intestinal involvement also had a resection, but died immediately postoperatively. Resection rates were similar between South Asians and Europeans $\left(\chi^{2}=0.06 \mathrm{~ns}\right)$.

\section{MORTALITY}

Seven patients died during the study period, two of whom were European. The expected number of deaths among South Asians was $7 \cdot 4$ (standardised mortality ratio $=68,95 \%$ confidence interval 20-160) compared with 0.27 among Europeans (standardised mortality ratio $=755,95 \%$ confidence interval 90-2730, $\chi^{2}=11 \cdot 4, \mathrm{p}<0.001$ ). 
DELAY IN DIAGNOSIS

Among the South Asians there were reliable data for 49 cases with a mean time from onset to diagnosis of 30 weeks (SD (40)). There was no comparable information for Europeans.

\section{Discussion}

The standardised incidence of abdominal tuberculosis in South Asians in Leicester fell significantly during the study period and is now $9 \cdot 2$ cases $/ 10^{5} /$ year as has the relative risk to South Asians. This is the first time a decline in the incidence of abdominal tuberculosis in South Asians has been shown and it recalls the changes seen with pulmonary tuberculosis in Britain during the 1940s.

The majority of cases of abdominal tuberculosis arose among immigrants rather than their offspring. This may suggest that the falling incidence is related to a reduction in numbers of immigrants. The majority of migrants to Leicestershire arrived in the late 1960s and early 1970s. ${ }^{10}$ "About half came from East Africa." The age specific incidence is highest among those aged between 20 and 60 years, however, and few second generation South Asians had reached this age during the study period. Those born after the 1967 migration from Kenya would only have been 22 years old at the end of the study and it is clearly too early to accurately assess the risk to the second generation.

This is the first attempt to investigate mortality from abdominal tuberculosis in a community based study. The standardised mortality ratio surprisingly was not increased. The time taken from onset of symptoms to diagnosis was relatively short. Together these data suggest that clinicians have a high index of suspicion, treatment is effective, and prognosis good. The two deaths among six European patients appears poor, although the wide confidence intervals shows the limited value of this observation although clinicians should be vigilant to this differential diagnosis in Europeans.

The incidence of abdominal tuberculosis in South Asians in Leicester is similar to five cases/ 105/year reported by Palmer et $a l^{2}$ between 1973 and 1983 in West London. In Bradford 45 cases (1967-77) were identified among a population of 36000 South Asians. $^{3}$ Although the incidence was not calculated it was probably about 12.5 cases/105/year. In Blackburn 109 cases were identified with an incidence of 0.43 cases $/ 10 \%$ year in Europeans and 35.7 cases $/ 105 /$ year in South Asians between 1970 and $1984 .{ }^{4}$ These disparities among South Asians are unlikely to simply reflect a continuing downward trend and may suggest different susceptibilities between ethnic groups. For example, pulmonary tuberculosis is known to be seven times more common in Hindus than Muslims.' The authors suggest that Muslims are less likely to be vegetarian than Hindus and this may affect resistance to infection.'

The differentiation of Crohn's disease from abdominal tuberculosis may be difficult. ${ }^{5-8}$ When the peritoneum is infected analysis of ascites is often characteristic. ${ }^{23}$ Abdominal tuberculosis can, however, mimic Crohn's disease of the terminal ileum, ${ }^{5}$ jejunum, ${ }^{6}$ and colon. ${ }^{78}$ While the demonstration of caseating necrosis or the culture of acid alcohol fast bacilli are necessary and are the yard sticks against which other investigations have to be measured, characteristic features may be found by endoscopy or radiology. ${ }^{24}$ Tandon compared abdominal tuberculosis and Crohn's disease in Indian patients and found little difficulty in distinguishing the conditions histologically; caseation and confluent granulomas were found in abdominal tuberculosis, while in Crohn's disease 'transmural cracks and fissures were consistently observed.' ${ }^{25}$ A therapeutic trial of antituberculous therapy has been suggested in equivocal cases where there is sufficient clinical suspicion. ${ }^{26}$

A parallel investigation of Crohn's disease in South Asians in Leicester ${ }^{27}$ has shown Muslims to have a similar incidence to Europeans, but Hindus and Sikhs significantly less Crohn's disease. This pattern of susceptibility is the reverse of that in abdominal tuberculosis. It is possible that some cases of abdominal tuberculosis were actually Crohn's disease, the converse is less likely as each case met internationally accepted diagnostic criteria. The time taken to make the diagnosis of abdominal tuberculosis was similar to that taken to diagnose Crohn's disease in South Asians, ${ }^{27} 22 \cdot 8$ weeks ( $t=$ $1 \cdot 27 \mathrm{~ns})$.

Clinicians need to be aware of the changing incidence of abdominal tuberculosis and Crohn's disease in South Asians. Abdominal tuberculosis is still common and in view of the possible adverse effects of inappropriate treatment its distinction from Crohn's disease is essential. The diagnosis can no longer be simply based on a patients' ethnic origin. There is no place for complacency; abdominal tuberculosis remains a significant problem in some communities in the United Kingdom.

We wish to thank the British Digestive Foundation and the National Association for Crohn's and Colitis who supported Dr V Jayanthi during this programme and the Hilden Trust who provided similar support for Dr C Probert. We wish to thank the provided similar support for Dr C Probert. We wish to thank the giving us access to patients under their care.

1 Finch PJ, Millard FJC, Maxwell JD. Risk of tuberculosis in immigrant Asians: culturally acquired immunodeficiency? immigrant Asians: cult

2 Palmer KR, Patil DH, Basran GS, Riordan JF, Silk DBA. Abdominal tuberculosis in urban Britain - a common Abdominal tuberculosis in urban
disease. Gut 1985; 26: 1296-305.

3 Findlay JM, Addison NV, Stevenson DK, Mirza ZA. Tuberculosis of the gastrointestinal tract, 1967-77. F $R$ Soc Med 1979; 72: 587-90.

4 Klimach OE, Ormerod LP. Gastrointestinal tuberculosis: a retrospective review of 109 cases in a district general hospital. OF Med 1985; 56: 569-78.

5 Burke GJ, Zafar SA. Problems in distinguishing tuberculosis of bowel from Crohn's disease in Asians. BMF 1975; 4: 395-7.

6 Humphreys C, Wake PN, Walker R. Jejunoileal tuberculosis; a diagnostic pitfall in Crohn's disease. BMF 1980; 3: 118-9.

7 Devanesan JD, Sable RA, Pitchumoni CS, Lev R, Zapiach L. Segmental tuberculosis of the colon mimicking carcinoma.

8 Carr-Locke DL, Findlay DBL. Radiological demonstration of colonic aphthoid ulcers in a patient with intestinal tuberculosis. Gut 1983; 24: 453-5.

9 Jayanthi V, Probert CSJ, Mayberry JF. Incidence of Crohn's disease in Leicester, 1972-89. Gut 1991; 32: A579.

10 Marett V. Immigrants Settling in the City. Leicester: Leicester University Press, 1989.

11 Survey of Leicester 1983. Leicester City Council and Leicestershire County Council.

12 Carey S, Shukur A. A profile of the Bangladeshi community of East London. New Community 1985; 12: 405-16. 
13 Ballard R, Ballard C. The Sikhs: The development of South Asian settlements in Britain. In: Watson JL, ed. Between two cultures. Oxford: Blackwell, 1977.

14 Office of Population Censuses and Surveys. Registrar Generals statistical reviews of England and Wales 1971. London: HMSO, 1971.

15 Office of Population Censuses and Surveys. 1981 OPCS Census county reports Leicestershire. London: HMSO 1982.

16 Leicester Council for Community Relations Annual Report 1982.

17 Langman MJS. The epidemiology of chronic digestive disease. London: Edward Arnold, 1979.

18 Probert CSJ, Jayanthi V, Pinder D, Wicks AC, Mayberry JF. An epidemiological study of ulcerative proctocolitis in Indian migrants and the indigenous population of Leicestershire, 1972-1989. Gut 1992; 33: 687-93.

19 Armitage P, Berry G. Statistic methods in medical research Oxford: Blackwell, 1987.

20 Gardner MJ, Altman DG, eds. Statistics with confidence. London: BMJ, 1989.
21 Office of Population Censuses and Surveys. Mortality statistics (area). London: HMSO, 1974 to 1989 (16 editions).

22 Marmot MG, Adelstein AM, Bulusu L. Immigrant mortality in England and Wales, 1970-78. London: HMSO, 1984

23 Bastani B, Shariatzadeh MR, Dehdashti F. Tuberculous peritonitis - report of 30 cases and review of the literature. Qf Med 1985; 56: 549-57.

24 Aoki G, Nagasako K, Nakae Y, Suzuki H, Takemoto T. The fibrecolonoscopic diagnosis of intestinal tuberculosis.

25 Tandon HD, Prakash A. Pathology of intestinal tuberculosis and its distinction from Crohn's disease. Gut 1972; 13:

26 Silk DBA, Palmer KR. Abdominal tuberculosis. Gastroenterol Practice 1987; 1: 6-10.

27 Jayanthi V, Probert CSJ, Pinder D, Wicks AC, Mayberry JF. Epidemiology of Crohn's disease in Indian migrants and the indigenous population of Leicestershire. (Submitted for publication). Qf Med 1992; 82: 125-37. 